\section{Suppression of PV Output Fluctuation Using a Battery Energy Storage System with Model Predictive Control}

\author{
Dwi Riana Aryani ${ }^{1}$, Jung-Su Kim ${ }^{2}$, and Hwachang Song ${ }^{2}$ \\ ${ }^{1}$ Department of Electrical Engineering, University of Indonesia, Depok, Jawa Bart, Indonesia \\ ${ }^{2}$ Department of Electrical and Information Engineering, Seoul National University of Science \& Technology, \\ Seoul, Korea
}

\begin{abstract}
This paper provides a control strategy for battery energy storage system (BESS) in suppressing the photovoltaic (PV) output fluctuation so that the smoothed power delivered to the grid satisfies the maximum ramp requirement of the system. As one of robust control solutions, model predictive control (MPC) is proposed so the control actions of battery charging and discharging can be planned by considering some constraints such as battery power and state of charge (SoC) limitations. Moving average filter (MAF) is used to generate the output reference for the combined system of PV and BESS where the time window is selected by considering the maximum ramp provided to the system. The simulation results show that the proposed control strategy is able to track the prescribed reference effectively so the smoothing strategy meets the fluctuations limit while satisfying the battery constraints.
\end{abstract}

Keywords: Battery energy storage systems (BESS), Fluctuation suppression, Model predictive control (MPC), Moving average filter (MAF), Photovoltaic (PV) systems

\section{Introduction}

In recent years, utilization of photovoltaic (PV) systems as one of renewable energy sources (RES) has been rapidly spreading throughout the world due to its easy installation. One of common issues on PV systems as similar with other kinds of RES are output stabilization because of its high dependence on climate conditions such as solar radiation and weather temperature. The output fluctuation makes PV not reliable because it might cause power imbalance and frequency deviation.

To alleviate the impacts of the PV fluctuation on system reliability, system operators (SO) apply a certain limit of the fluctuations to be delivered to the grid. The requirements of fluctuations limit are difference for each SO, normally ranging from $1 \% / \mathrm{min}$ to $10 \% / \mathrm{min}$ in terms of the rated power [1]. Therefore, plant owners need to devise countermeasures to suppress PV output fluctuations within the allowable limits. Installation of battery energy storage systems (BESS) is one of the options for output stabilization. Beside fluctuation suppression, integrating BESS with RESs also can achieve other objectives, such as capacity firming and peak shaving [2, 3].

Optimal smoothing strategies for wind farms and PV farms using BESS have been proposed in [4-6]. For the purpose, this paper also employs moving average filter (MAF) to generate the 
output power reference of the combined system of PV and BESS. To fulfill the fluctuations limits, the battery size and the time window of MAF are determined by considering the maximum ramp rate [7].

This paper proposes the application of one-step model predictive control (MPC) for the BESS charging and discharging algorithms. The reason of selecting MPC is that it can provide more benefits compared to other controllers [8, 9]. MPC is able to consider the predicted PV system performance and hence to schedule the control action with the anticipated PV output power ramps. For the purpose, an accurate model of BESS is required so that the control actions can deal with the BESS physical limits such as BESS power and state of charge (SoC). A one-step horizon is chosen for faster computation since MPC recursively computes for an optimal control solution over the whole prediction horizon [10-[12].

This paper proposes an MPC-based control strategy for BESS operation in such a way that the maximum fluctuations of the smoothed PV plant output should satisfy the requirement from SO. The selection of window size of MAF is also given in order to generate the reference of the plant outputs. In addition, this paper provides an estimation of sizing the required BESS capacity for the fluctuation level with the given characteristics.

In the paper, the proposed strategy can provide the following advantages: i) one-step MPC for controlling battery power can satisfy the defined ramp rate requirement considering physical constraints; ii) The solution of the MPC problem is obtained with a closed form and hence then fast computation is possible; iii) By including the selection of window size of MAF and the estimation of the battery size, the battery control strategy with one-step MPC is able to suppress the plant outputs satisfying the fluctuation regulation while fulfilling the physical constraints of the BESS.

This paper is organized as follows: the problem formulation consisted of the system model, physical constraints of BESS, and PV output fluctuation is presented in Section 2. The proposed method of one step MPC for controlling battery output as well as calculations of window size of MAF and the battery size are described in Section 3. Simulation results of the proposed method by varying the window size of MAF and the battery size is described in Section 4. And the conclusion is presented in Section 5.

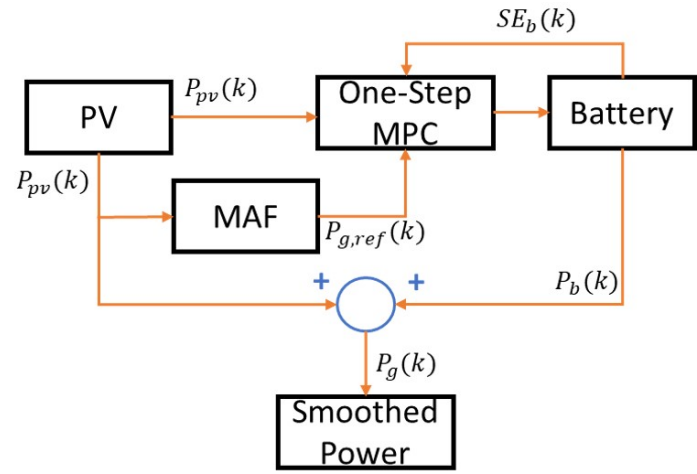

Figure 1. Block diagram of the smoothed power delivered to the grid output.

\section{Problem Formulation}

The structure of the smoothed PV farm output control is shown in Figure 1. The fluctuating PV output is compensated by the battery, so that the integration of PV and battery delivers a smoothed power to the grid. The reference of the smoothed power is generated by utilizing the moving average filter to the PV output, where the window size is set in such a way that the defined maximum ramp rate is satisfied. For the purpose, an MPC control is employed in order to obtain optimal charging and discharging of the battery power.

\subsection{System Model}

At time $(k)$, power that will be injected to the grid $P_{g}(k)$ is obtained by smoothing the PV output $P_{p v}(k)$ using battery power $P_{b}(k)$ as described in (1).

$$
P_{g}(k)=P_{p v}(k)+P_{b}(k) .
$$

The output of battery is expected to drive the combined PV and battery outputs meet the fluctuations allowed by the SO. Hence, the plant model is given as:

$$
\begin{aligned}
\underbrace{\left[\begin{array}{c}
S E_{b}(k+1) \\
P_{b, p r e v}(k+1)
\end{array}\right]}_{x(k+1)}= & \underbrace{\left[\begin{array}{cc}
1 & 0 \\
0 & 0
\end{array}\right]}_{A} \underbrace{\left[\begin{array}{c}
S E_{b}(k) \\
P_{b, \text { prev }}(k)
\end{array}\right]}_{x(k)} \\
& +\underbrace{\left[\begin{array}{c}
-t_{d} \\
1
\end{array}\right]}_{B} \underbrace{\left[P_{b}(k)\right]}_{u(k)},
\end{aligned}
$$




$$
\begin{aligned}
\underbrace{\left[\begin{array}{c}
P_{g}(k+1) \\
\Delta P_{g}(k+1)
\end{array}\right]}_{y(k)} & =\underbrace{\left[\begin{array}{cc}
0 & 0 \\
0 & -1
\end{array}\right]}_{C} \underbrace{\left[\begin{array}{c}
S E_{b}(k) \\
P_{b, p r e v}(k)
\end{array}\right]}_{x(k)} \\
& +\underbrace{\left[\begin{array}{c}
1 \\
1
\end{array}\right]}_{D_{u}} \underbrace{\left[P_{b}(k)\right]}_{u(k)} \\
& +\underbrace{\left[\begin{array}{cc}
1 & 0 \\
0 & 1
\end{array}\right]}_{D_{w}} \underbrace{\left[\begin{array}{c}
P_{p v}(k) \\
\Delta P_{p v}(k)
\end{array}\right]}_{w(k)},
\end{aligned}
$$

where $S E_{b}(k)$ denotes the stored energy at $k$, and $P_{b, p r e v}(k)$ stands for the battery power at the previous step from $k$. In (3), $\Delta P_{p v}(k)$ represents the change of PV outputs at $k-1$ and $k$.

Smoothing the PV output means that the ramping needs to be minimized such that the energy fluctuations delivered to the grid will not exceed the maximum allowable limit. By considering the power delivered to the grid in (1), it is important to set the reference of the delivered power $P_{g, r e f}$ and design the battery sizing $P_{b}$ properly. $P_{g, r e f}$ needs to fulfill the requirement of the maximum allowable fluctuation from SO. While in order to meet $P_{g, r e f}$, battery would charge the excessed energy or discharge the required power. However, battery has some constraints that would resist the system to achieve its control objective, for example the state of charge (SoC) and battery power rating constraints.

\subsection{Physical Constraints of BESS}

As described in previous section, SoC and power rating are considered as physical constraints of the battery. In this paper, the $\mathrm{SoC}$ model in discrete-time model is given by:

$$
S E_{b}(k+1)=S E_{b}(k)-t_{d} P_{b}(k),
$$

where $t_{d}$ is the time coefficient representing the conversion of battery power to energy, and $P_{b}$ is the battery output power. Taking the battery physical limitations into account, $S E_{b}(k)$ and $P_{b}(k)$ should satisfy the following constraints.

$$
\begin{aligned}
& P_{b, \min } \leq P_{b}(k) \leq P_{b, \max }, \\
& S E_{b, \min } \leq S E_{b}(k) \leq S E_{b, \max } .
\end{aligned}
$$

In order to cope with this problem, one-step MPC is employed as the control strategy for the battery such that the battery output would lead the $P_{g}(k)$ to the desired reference $P_{g, r e f}(k)$ satisfying the constraints.

\subsection{Smoothing PV Output Fluctuation}

As other RESs, power outputs of PV systems depend on the climate conditions of the installed locations, and the changes in temperature and irradiation cause the fluctuation of PV output power. Such makes PV not reliable because it might cause power imbalance and frequency deviation. Thus, SOs applies constraints to output fluctuations from PV plants, normally ranging from $1 \% / \mathrm{min}$ to $10 \% / \mathrm{min}$. Using BESS can improve the ramp rate control performance. In this paper, a time series of PV output power was directly employed to simulate PV output fluctuation.

The main constraint for ramp-rate control is indeed to satisfy the given criterion, which is given as follows:

$$
\min \sum\left(P_{b}(k)\right)^{2}
$$

The main constraint for ramp-rate control is indeed to satisfy the given criterion, which is given as follows:

$$
\left|P_{g}(k)-P_{g}(k-1)\right|=a S_{P V},
$$

where $S_{P V}$ denotes the rated power of the PV system and $a$ stands for the allowable percent ratio to $S_{P V}$ in the criterion. Also the control problem includes the system model equations from (1) to (6). To realize the control effectively, adequate determining the reference value for the output is crucial, and the details on the realization are given in the next chapter.

\section{One Step MPC Strategy with MAF Consider- ing Battery Size}

\subsection{Moving Average Filter}

In this paper, MAF is used to smooth PV outputs by generating the reference of plant output at $k$ time period $P_{g, r e f}(k)$ as:

$$
P_{g, r e f}(k)=\frac{1}{T} \sum_{n=k-T+1}^{k} P_{p v}(n),
$$

where $P_{p v}(n)$ denotes PV output at $n$, and $T$ stands for the size of the applied moving window. The plant output is combined with the PV system and BESS.

Since the primary control objective is to suppress the fluctuation to the allowable ramp limits, the ramping of the $P_{g, r e f}(k)$ needs to satisfy the defined maximum ramp rate. It is assumed that the maximum generation capacity of $\mathrm{PV}$ farm is $P_{p v, \max }$ and the ramp maximum for $P_{g, \text { ref }}$ over a certain period is $r_{\max }$, 
which is given by the operator. The window size for the needed MAF, $T$, can be determined by (13) in order to satisfy the maximum ramp limit.

$$
\begin{aligned}
& r_{\text {max }}=\max \left(\left\|P_{g, r e f}(k)-P_{g, r e f}(k-1)\right\|\right), \\
& r_{\text {max }}=\max \left(\frac{1}{T}\left\|P_{p v}(k)-P_{p v}(k-T)\right\|\right), \\
& \left\|P_{p v}(k)-P_{p v}(k-T)\right\| \leq P_{p v, \text { max }}, \\
& T \leq \frac{P_{p v, \text { max }}}{r_{\max }} .
\end{aligned}
$$

\subsection{Battery Size}

After generating the reference of the smoothed PV output power $P_{g, r e f}$, battery power rating can be determined. The ramp of PV output, battery power, and the plant output are defined as follows:

$$
\begin{aligned}
& \Delta P_{p v}(k)=P_{p v}(k)-P_{p v}(k-1), \\
& \Delta P_{b}(k)=P_{b}(k)-P_{b}(k-1), \\
& \Delta P_{g}(k)=P_{g}(k)-P_{g}(k-1) .
\end{aligned}
$$

Considering (1), $\Delta P_{g}$ can be determined by

$$
\Delta P_{g}(k)=\Delta P+p v(k)-\Delta P_{b}(k)
$$

Battery size needs to be properly selected in such a way that the requirement of the maximum fluctuation in the system is fulfilled. In order to achieve that, the battery rating has to be equal to or larger than the difference between maximum ramping of $\mathrm{PV}$ output $\Delta P_{p v, \max }$ and the maximum ramping of the smoothed power $\Delta P_{g, \max }$ as follows:

$$
\begin{aligned}
& P_{g} \leq \Delta P_{g, \text { max }}, \\
& P_{b} \geq \Delta P_{p v, \text { max }}-\Delta P_{g, \text { max }} .
\end{aligned}
$$

\subsection{One-Step MPC for Battery Output}

In order to charge and discharge battery optimally, the cost function of the battery output $J(u(k))$ needs to be minimized as follows:

$$
J(u(k))=\alpha\left(y(k)-y_{r e f}(k)\right)^{2}+\beta(u(k))^{2},
$$

where the battery output is defined as the control input $u(k)$ at sampling time $k$, while $\alpha$ and $\beta$ are the sampling parameters [12]. According to 33, $y(k)$ represents $P_{g}(k)$ and $\Delta P_{g}(k)$. However, according to (17), $P_{g}(k)$ is directly proportional to
$\Delta P_{g}(k)$ and it is assumed that $P_{g}(k)$ is able to be driven to $P_{g, r e f}(k)$ so $\Delta P_{g}(k)$ would automatically follow $\Delta P_{g, r e f}(k)$ as well. Thus, in this paper, to formulate the one-step MPC for controlling battery power output, $y(k)$ is considered to only represent $P_{g}(k)$.

The optimal input of (18) without taking the constraints in (5) and (6) into account, $\hat{u}(k)$, is as follows:

$$
\widehat{u}(k)=\frac{\alpha\left(P_{p v}(k)-P_{g, r e f}(k)\right)}{(\alpha+\beta)} .
$$

While the optimal input by considering such constraints, $u^{*}(k)$, is given by:

$$
u^{*}(k)= \begin{cases}u_{a}, & \text { if } \widehat{u}(k)>u_{a}, \\ \widehat{u}(k), & \text { otherwise, } \\ u_{b}, & \text { if } \widehat{u}(k)<u_{b},\end{cases}
$$

where

$$
\begin{aligned}
& u_{a}=\min \left\{u_{\max }, \frac{S E_{b}(k)-S E_{b, \min }}{t_{d}}\right\}, \\
& u_{b}=\max \left\{u_{\min }, \frac{S E_{b}(k)-S E_{b, \max }}{t_{d}}\right\} .
\end{aligned}
$$

\section{Simulation Results}

The simulations in this paper are done in MATLAB $2016 \mathrm{~b}$. The PV output data is taken for 15 hours, from 5 am to $8 \mathrm{pm}$. The PV system capacity is around $10 \mathrm{MW}$. Figure 2 shows the measured PV output $P_{p v}$. The reference of the smoothed PV output $P_{g, r e f}$ is generated from the measured PV output data $P_{p v}$ by using MAF with time window 10 minutes or 600 seconds. This value is determined according to Eq. (11) where $P_{p v, \max }$ is $10 \mathrm{MW}$. The maximum ramping of $P_{g}$ allowed by the SO is $10 \% / \mathrm{min}$ of the $\mathrm{PV}$ rating or around $1 \mathrm{MW} / \mathrm{min}$.

In order to keep $P_{g}$ close to the determined $P_{g, r e f}, P_{p v}$ needs to be used for the MPC scheme to provide the battery power $P_{b}$, which is the control input. That is, $P_{b}$ is scheduled to charge or discharge energy from or to the grid in such a way that the fluctuations of $P_{g}$ should not violate the required ramp limit given by the SO. Based on Eq. (17), the battery power rating should consider the maximum $\mathrm{PV}$ output ramping and the maximum smoothed PV output ramping. The maximum PV output ramping $\left|\Delta P_{p v, \max }\right|$ happened at 1:25 pm (the 505th sample) around 6.7 MW as shown in Figure 3, while the allowed maximum smoothed power ramping is $1 \mathrm{MW} / \mathrm{min}$. Thus, the power rating of battery is at least around $5.7 \mathrm{MW}$, with \pm 5.7 


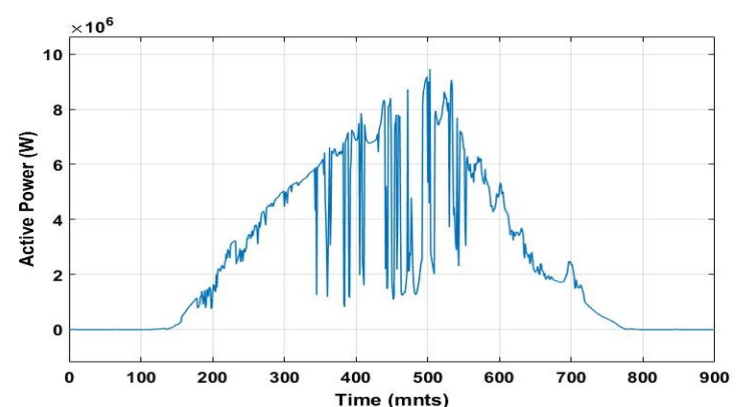

Figure 2. The measured PV output $P_{p v}$.

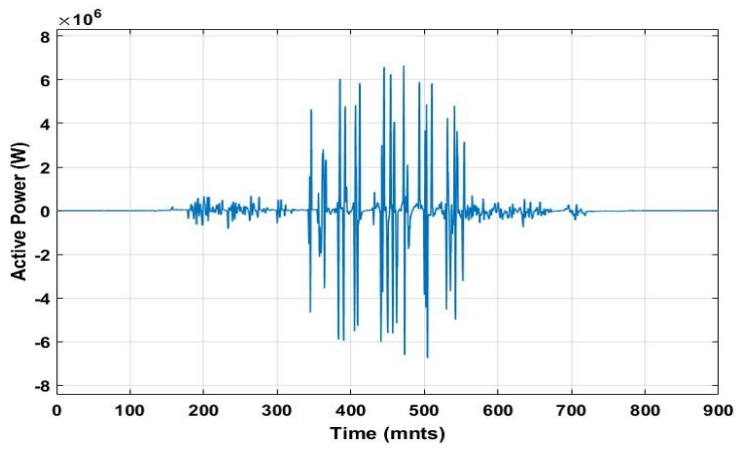

Figure 3. PV output ramping.

MW as the maximum charging/discharging. It is assumed that the capacity rate of the battery is 1 , which means that the maximum stored energy is 5.7 MWh.

Given the MAF and the battery power rating as determined above, by utilizing the proposed one step MPC, the comparison between the smoothed PV output of the combined BESS and PV system $\left(P_{g}\right)$ and its reference $\left(P_{g, r e f}\right)$ is shown in Figure 4. In this simulation, $\alpha$ and $\beta$ are set to 0.95 and 0.05 for the objective function for the MPC. Figure 5 illustrates the battery charging and discharging power $\left(P_{b}\right)$, which are considered as the control input. Figure 6 displays the battery stored energy $\left(S E_{b}\right)$ and the battery power at $k-1\left(P_{b, p r e v}\right)$ during the simulation. In Figure 6, the positive value represents the discharging mode while the negative one does the charging mode. Figure 7 shows the fluctuation of the smoothed PV output per minute.

From Figures 2-7, one can notice that the smoothed PV output power is able to satisfy the requirement of the maximum fluctuation $10 \% / \mathrm{min}$. In other words, the ramping of the smoothed PV output is successfully maintained below 1 $\mathrm{MW} / \mathrm{min}$. Besides, the battery power always satisfies the SoC and power rating constraints. From 5 am to 3 pm (1st-600th samples), battery has more charging phases, and hence the energy increases during that period. While at $3 \mathrm{pm}$ to $8 \mathrm{pm}$

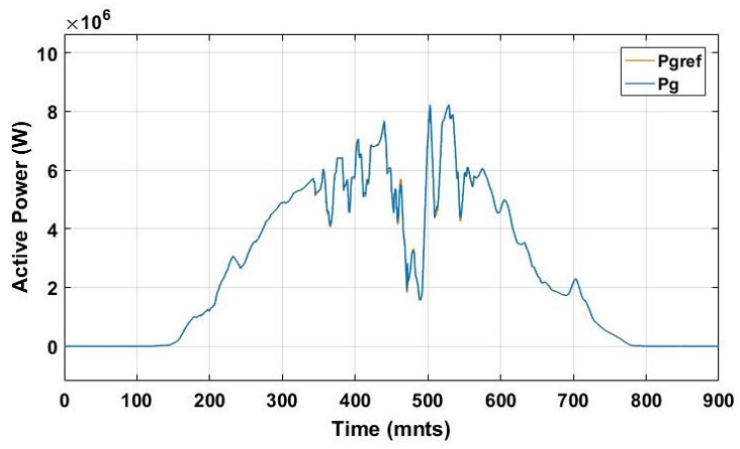

Figure 4. Comparison between $P_{g}$ (blue line) and $P_{g, r e f}$ (orange line).

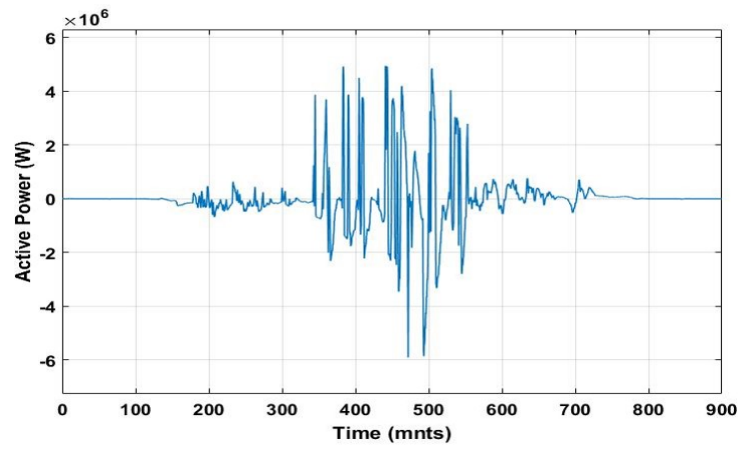

Figure 5. Battery charge and discharge power $P_{b}$.

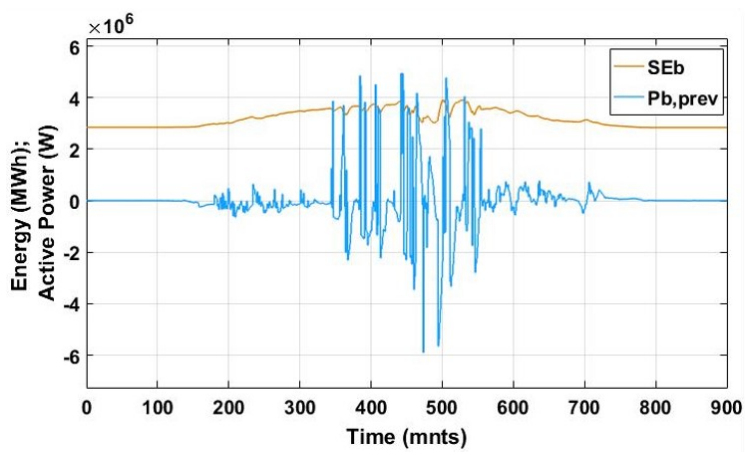

Figure 6. Battery energy $S E_{b}$ and battery power at $k-1\left(P_{b, p r e v}\right)$.

(601st-900th samples), battery has more discharging phases, so the energy decreases. During the whole periods of operation, the battery energy was kept maintained close to $50 \%$ SoC, around 50\%-65\%. This operating range of SoC implies that the charging and discharging phases of the battery has been optimized. Moreover, by maintaining such SoC range of battery operation, it might help to prolong the battery lifetime. It is concluded that the proper selection of time window and battery rating, and utilizing one-step MPC for controlling battery charge and discharge phases would provide an optimal fluctuation suppression strategy. 


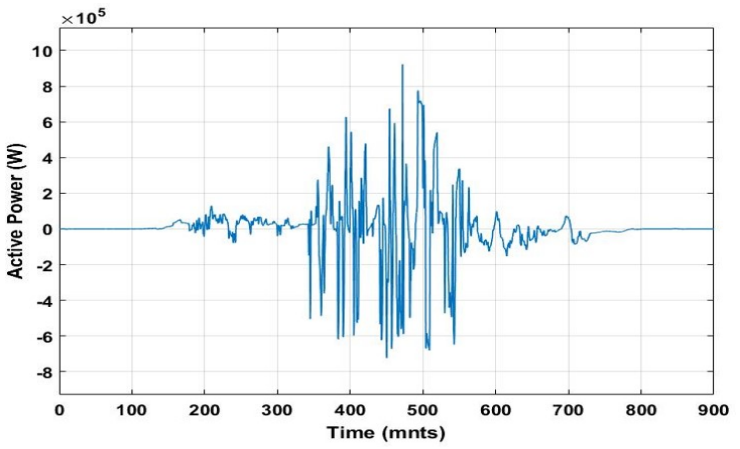

Figure 7. Fluctuation of the smoothed PV output with 1 minute sampling time $\Delta P_{g}$.

Another simulation results are illustrated in Figures 8 and 9 by varying the time window and battery size beyond the proposed value as comparison with the proposed optimal values.

In Figure 8, the battery power rating is selected as the proposed size, around 5.9 MW. Besides, the MAF time window is set larger than the proposed value, around 33 minutes. In Figure 9(a), with the selected time window, the reference of the smoothed power $P_{g, r e f}$ is getting smoother. However, utilizing one step MPC for controlling battery power by considering the constraints with the selected large time window makes the battery charge and discharge power reaching the limit of $P_{b \min }$, $P_{b \max }, S E_{b \min }$, and $S E_{b, \max }$ quickly. Hence, the battery control strategy could not satisfy its physical constraints and at $\mathrm{t}=$ 9:50 am (290th samples) the generated $P_{g}$ started to not follow the reference. Eventually, the fluctuation of $P_{g}$ does not fulfill the requirement from $\mathrm{SO}$ at 11:30 am, 11:40 am, 12:20 pm, and 1:50 pm (390th, 400th, 440th, and 530th samples).

The next simulation is done with the battery power rating was set below the proposed size, around $4.9 \mathrm{MW}$, as shown in Figure 9. The MAF time window is within the proposed value, around 10 minutes. Since the battery rating is below its capacity to charge or discharge power while satisfying the $P_{g, r e f}$, battery power and stored energy reached its minimum and maximum limits quickly. Eventually, the fluctuation of $P_{g}$ is beyond its limit at $1: 10 \mathrm{pm}$ and 1:20 pm (490th and 500th samples). From simulations above, it can be concluded that the proper selection of the time window and the battery rated power, and by utilizing one-step MPC for controlling battery charge and discharge phases would successfully provide an optimal fluctuation suppression strategy.

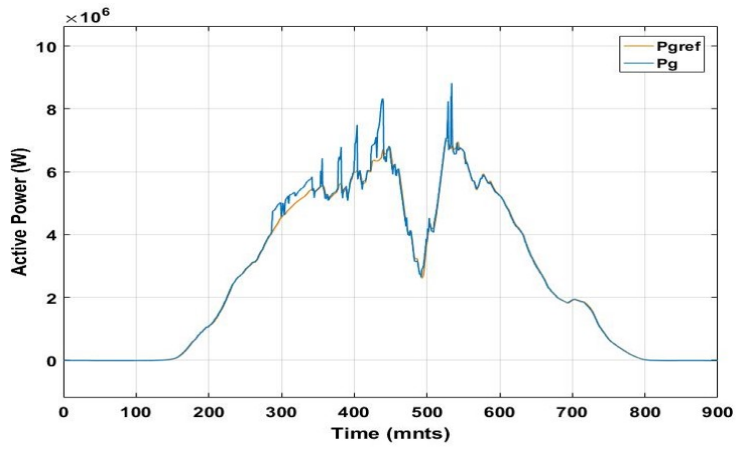

(a)

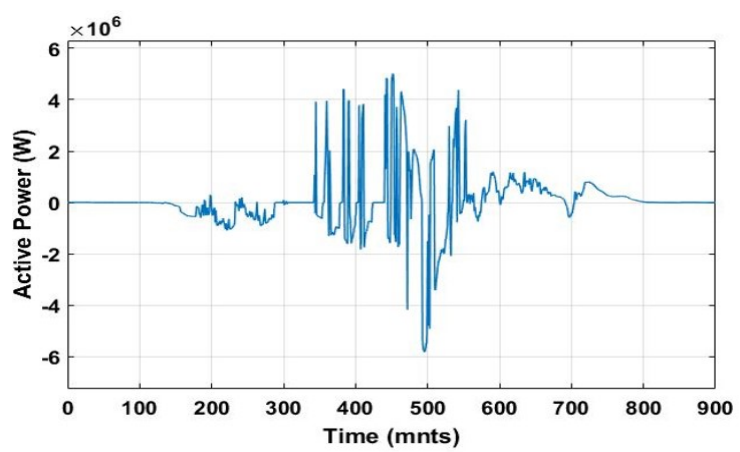

(b)

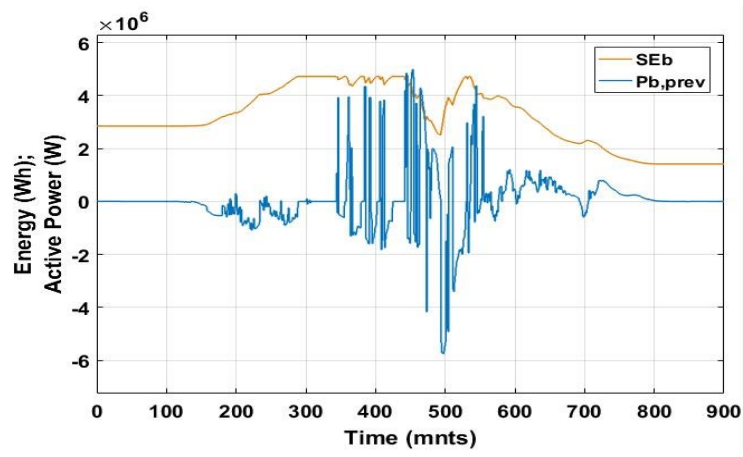

(c)

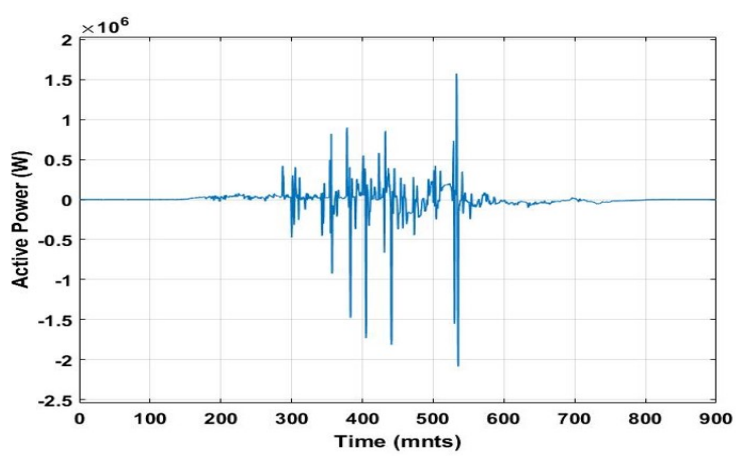

(d)

Figure 8. Control performance of PV output fluctuation suppression using battery power rated $5.9 \mathrm{MW}$ and MAF time window 33 minutes. (a) Comparison between $P_{g}$ and $P_{g, r e f}$, (b) control input $P_{b}$, (c) controller states $S E_{b}$ and $P_{b, p r e v}$, (d) $\Delta P_{g}$. 


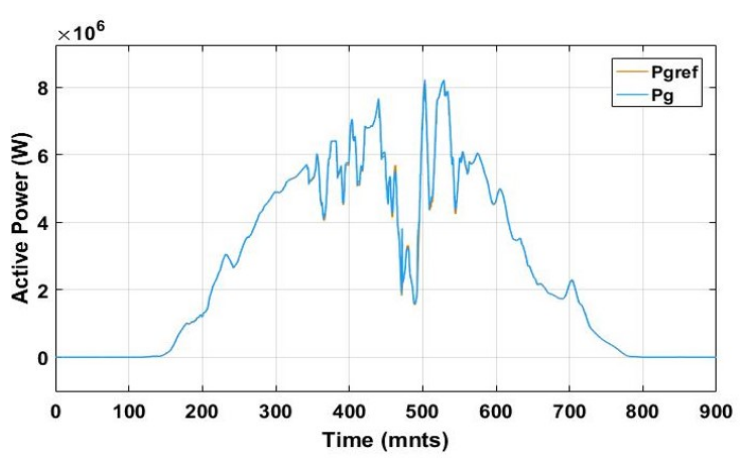

(a)

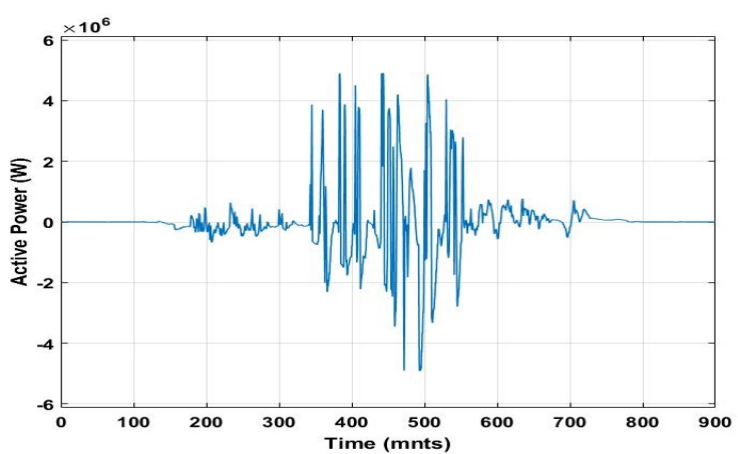

(b)

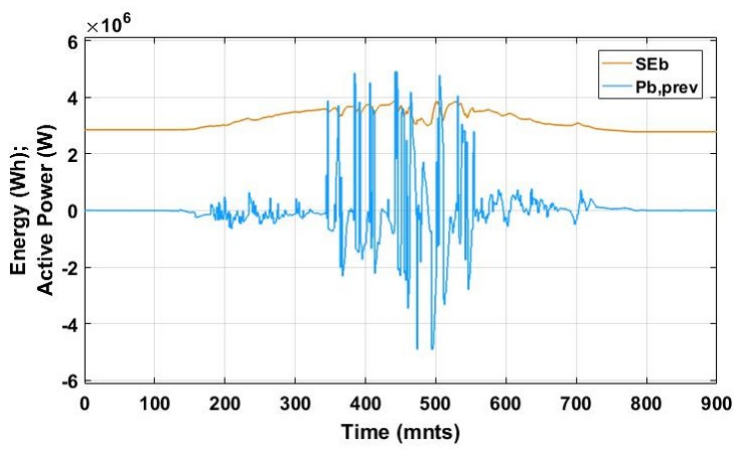

(c)

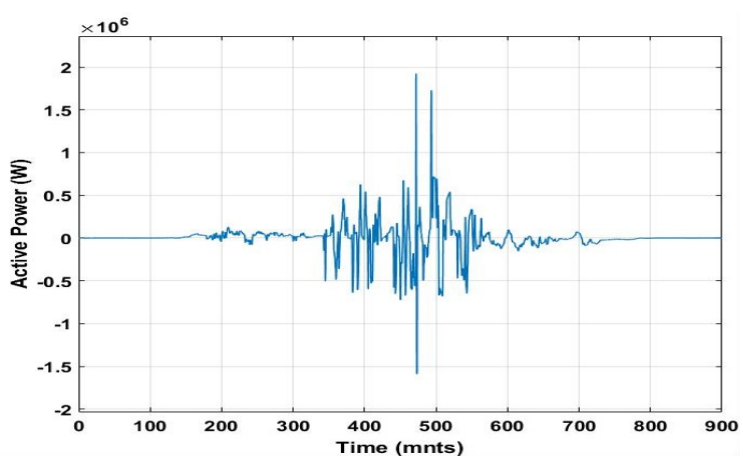

(d)

Figure 9. Control performance of PV output fluctuation suppression using battery power rated $4.9 \mathrm{MW}$ and MAF time window 10 minutes. (a) Comparison between $P_{g}$ and $P_{g, r e f}$, (b) control input $P_{b}$, (c) controller states $S E_{b}$ and $P_{b, p r e v}$, (d) $\Delta P_{g}$.

\section{Conclusion}

Suppressing the fluctuation output of renewable energy sources is desirable for each RES generator, and the fluctuation level needs to be constrained by SOs in order for secure operation of power systems. This paper proposes a control strategy of battery power to suppress the fluctuation of PV outputs in such a way that the requirement of maximum acceptable fluctuation level is satisfied in the regulation. From the simulation results, it is noticed that the proposed strategy of controlling battery power using the one-step MPC combined with the proper selection of MAF time window size and the battery rating, can provide the ability to achieve the suppression fluctuation within the desirable range, while the battery physical constraints are also assured.

\section{Conflict of Interest}

No potential conflict of interest relevant to this article was reported.

\section{Acknowledgements}

This work has been supported by Seoul National University of Science \& Technology.

\section{References}

[1] J. Marcos, I. de la Parra, M. Garcia, and L. Marroyo, "Control strategies to smooth short-term power fluctuations in large photovoltaic plants using battery storage systems," Energies, vol. 7, no. 10, pp. 6593-6619, 2014. https://doi.org/10.3390/en7106593

[2] S. Son and H. Song, "Real-time peak shaving algorithm using fuzzy wind power generation curves for large-scale battery energy storage systems," International Journal of Fuzzy Logic and Intelligent Systems, vol. 14, no. 4, pp. 305-312, 2014. https://doi.org/10.5391/IJFIS.2014.14.4. 305

[3] M. D. Robles, J. S. Kim, and H. Song, "Capacity firming for wind generation using one-step model predictive control and battery energy storage system," Journal of Electrical Engineering \& Technology, vol. 12, no. 5, pp. 2043-2050, 2017. https://doi.org/10.5370/JEET.2017.12 5.2043 
[4] A. Puri, "Optimally smoothing output of PV farms," in Proceeding of the IEEE PES General Meeting, National Harbor, MD, 2014, pp. 1-5. https://doi.org/10. 1109/PESGM.2014.6939029

[5] Y. Noro, S. Naoi, K. Toba, M. Kimura, T. Minegishi, M. Shimizu, S. Aoki, and Y. Okuda, "Power fluctuation suppression system for large scale PV," in Proceeding of the IEEE PES on Transmission and Distribution Conference and Exposition, Orlando, FL, 2012, pp. 1-6. https://doi.org/10.1109/TDC.2012.6281714

[6] R. Watanabe, Y. Ito, Y. Hida, R. Yokoyama, K. Iba, and T. Tsukada, "Optimal capacity selection of hybrid energy storage systems for suppressing PV output fluctuation," in Proceeding of the IEEE Innovative Smart Grid Technologies, Tianjin, China, 2012, pp. 1-5. https://doi.org/10.1109/ISGT-Asia.2012.6303135

[7] M. Akatsuka, R. Hara, H. Kita, T. Ito, Y. Ueda, and Y. Saito, "Estimation of battery capacity for suppression of a PV power plant output fluctuation," in Proceeding of the 35th IEEE Photovoltaic Specialists Conference, Honolulu, HI, 2010, pp. 540-543. https://doi.org/10.1109/ PVSC.2010.5616820

[8] D. Halamay, M. Antonishen, K. Lajoie, A. Bostrom, and T. K. A. Brekken, "Improving wind farm dispatchability using model predictive control for optimal operation of grid-scale energy storage," Energies, vol. 7, no. 9, pp. 5847-5862, 2014. https://doi.org/10.3390/en7095847

[9] D. Yang, J. Wen, K. W. Chan, and G. Cai, "Dispatching of wind/battery energy storage hybrid systems using inner point method-based model predictive control," Energies, vol. 9, no. 8, p. 629, 2016. https://doi.org/10.3390/ en9080629

[10] Y. Ma, Z. Miao, V. R. Disfani, and L. Fan, "A onestep model predictive control for modular multilevel converters," in Proceeding of the 2014 IEEE PES General Meeting, National Harbor, MD, 2014, pp. 1-5. https://doi.org/10.1109/PESGM.2014.6939209

[11] A. Balau and C. Lazar, "One step ahead MPC for an automotive control application," in Proceeding of the 2nd Eastern European Regional Conference on the Engineering of Computer Based Systems, Bratislava, Slovakia, 2011, pp. 61-70. https://doi.org/10.1109/ECBSEERC.2011.18
[12] Y. Shi, T. Chai, and H. Yue, "Robust one-step model predictive control for discrete time-delay systems," IFAC Proceedings Volumes, vol. 41, no. 2, pp. 61606165 , 2008. https://doi.org/10.3182/20080706-5-KR1001.01040

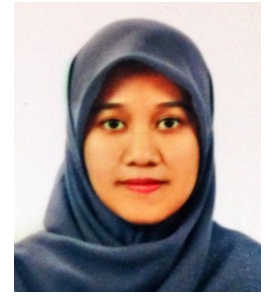

Dwi Riana Aryani received her B.S. degree in Electrical Engineering, University of Indonesia, in 2013. She received M.S. degree in Electrical and Information Engineering from Seoul Nat'l University of Science \& Technology, in 2017. Currently, she is working as a researcher in Electric Power and Energy Studies, in the Department of Electrical Engineering, University of Indonesia.

E-mail: dwiriana47@gmail.com

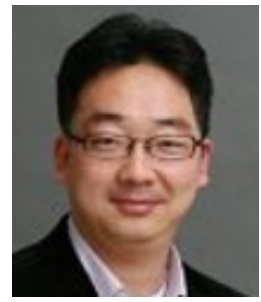

Jung-Su Kim received his B.S., M.S. and Ph.D. degrees in Electrical Engineering from Korea University. He was a postdoc at CDSL, Seoul National University, in 2005, at IST, University of Stuttgart, Germany from 2006-2007, and Systems Biology Laboratory, University of Leicester, UK, in 2008. Since 2009, he has been with the Department of Control and Instrumentation Engineering, Seoul National University of Science and Technology, as an assistant professor. His research interests include adaptive and predictive control, nonlinear control and systems biology.

E-mail: jungsu@ @eoultech.ac.kr

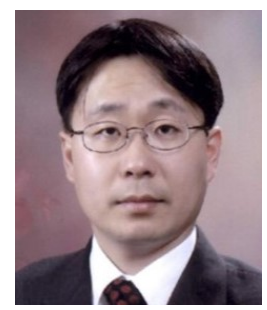

Hwachang Song received B.S., M.S. and $\mathrm{Ph} . \mathrm{D}$. in Electrical Engineering from Korea University in 1997, 1999 and 2003, respectively. He was a Post-doctoral Research Associate at Iowa State University from 2003 to 2004. He was working as a faculty member in the School of Electronic and Information Engineering, Kunsan National University, from 2005 to 2008. Currently, he is a Professor in the Department of Electrical and Information Engineering, Seoul Nat'l University of Science \& Technology. His research areas of interest are nonlinear optimization, power system stability and control, battery energy storage systems, and renewable energy resources.

E-mail: hcsong@seoultech.ac.kr 